\title{
Evaluation of Geological Risks of Commercial Development of Complex Structured Coal Deposits
}

\author{
Tamara Rogova ${ }^{1, *}$ and Sergey Shaklein ${ }^{2,3}$ \\ ${ }^{1}$ T.F. Gorbachev Kuzbass State Technical University, 650000, Kemerovo, Russia \\ ${ }^{2}$ Kemerovo branch of the Institute of Computational Technologies of Siberian Branch of the Russian \\ Academy of Sciences, 650025, Kemerovo, Russia \\ ${ }^{3}$ Federal State Budget Scientific Institution «The Federal Research Center of Coal and Coal \\ Chemistry of Siberian Branch of the Russian Academy of Sciences», 650065, Kemerovo, Russia
}

\begin{abstract}
The main risk in the commercial development of complex located coal deposits is a geological risk, which is assessed by the results of coal resources classification at sufficient confidence. In conditions of complex located coal deposits, the main factor which determines categories of coal Reserves is the confidence of geological structural data, a reliable estimation of which is provided only with the use of quantitative methods. The use of conventional geostatistical and geometrical approaches to the reserves categorization in this kind of deposits is impossible. The confidence of complex located coal deposits is proposed to be quantitatively evaluated by the similarity degree of seams position on the adjacent exploration lines. This similarity is estimated with a special similarity coefficient varying from 0 to 1 , determined based on curves correlation, which is adapted to the problem-solving method, in the complex plane. The obtained results provide the quality increase of the geological materials confidence evaluation and can be used to solve issues of the geological risk evaluation of the development of complex located coal deposits, during commercial or state expertise of geological information.
\end{abstract}

\section{Introduction}

The mining business, unlike other types of economic activity, is characterized by a very specific feature: its owner and investor have information about the equipment, technologies, current market conditions, etc., but never have unambiguous knowledge about in situ Mineral Resources, within which they carry out their activities. This leads to the emergence of a permanent additional risk factor, called geological risk, which is among the three of the most significant risks [1,2]. It is particularly pronounced in small-scale mining sectors [3] and in the open-pit process [4].

One of the main branches of the mining industry is coal one, which continues to have a significant role in the global economy. In the first 13 years of this century, coal production

${ }^{*}$ Corresponding author: $\underline{\text { rogovatb } @ \text {,kuzstu.ru }}$ 
in the world has increased as much as in the whole of the 20th century. During the period, the absolute increase in coal energy consumption amounted to almost half of its total global growth. None of the basic scenarios of global forecasts for the development of the global energy sector suggests a reduction in physical consumption of coal up to 2040. Coal needs are met not only by the mining of large deposits but also by small ones, including complex located deposits, which can be efficiently worked out only by the stripping mining method. The development of such deposits is carried out in the case of the presence of valuable coal mark in them when they locate near consumption areas and it's always associated with a high geological risk.

An example of complex located small deposits with resources of valuable coking coal is «Novobachatskiy» area located in the western part of the Kuznetsk Basin (figure 1). Despite the high density of the exploration grid (309 drill holes per sq.km), it was not possible to establish in detail the seam layers morphology features. As a result, the level of non-confirmation of coal reserves within the annual mining area is $41 \%$, which significantly exceeds the expected level.

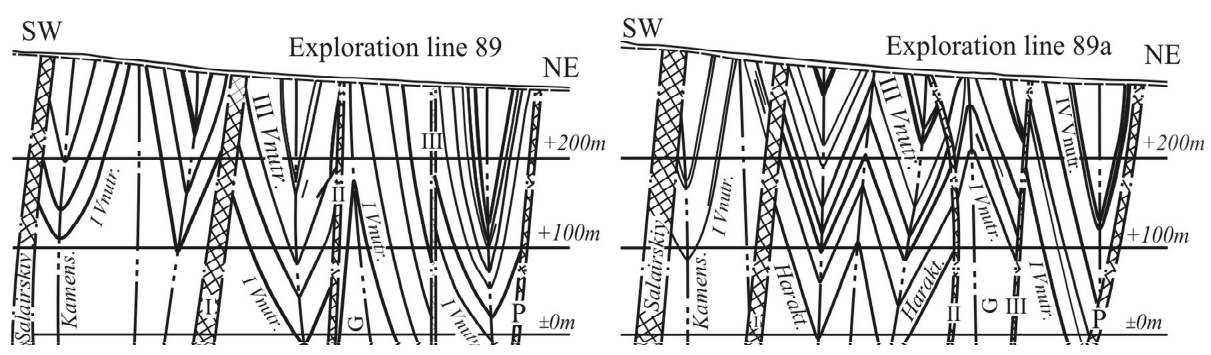

Fig. 1. Geological section of exploration line of the «Novobachatskiy» area, the distance between which is $100 \mathrm{~m}$.

The level of geological risk of commercial development of deposits is estimated by investors based on the results of the classification of resources according to categories of Mineral Reserves ("inferred", "indicated" and "measured"), performed in accordance with the requirements of the CRIRSCO public reporting codes [5]. Obviously, it is possible to single out only "inferred" and "indicated" coal reserves during the evaluation of such complex located deposits.

It's now recognized that a reliable assessment of categories of reserves is possible only through the use of quantitative methods. For the purpose, methods based on various geostatistical $[6,7,8]$ and geometric [9] approaches are used predominantly. It is generally recognized that in the conditions of coal deposits, the variography of variables is very difficult and carries the risk that the continuity of the variable may be overestimated, which is associated with the presence of closely located drill holes and their low quantity [10]. Under conditions of complex located coal deposits, the use of traditional geostatistical and geometric approaches is impossible at all.

Thus, at present, there are no quantitative methods for dividing the reserves of complex located coal deposits into "inferred" and "indicated" Coal Reserves, which makes the reliable estimation of the geological risk of their development impossible.

\section{Proposed method}

The model construction of a coal deposit always begins with an analysis of the geological section of exploration lines. The smaller the distance between two adjacent exploration lines, the more they are similar to each other. The "rate" of change in their similarity as the distance between sections increases at difficult deposits is higher than at simple ones. The 
degree of similarity of adjacent geological sections depends on the same factors as the confidence of the Mineral Reserve estimates, that is, on the density of the exploration lines and the complexity of the deposit geological structure and, accordingly, can be considered as a characteristic directly related to the confidence of the Mineral Reserve.

The approach proposed by S. A. Batugin to estimate the complexity of the geological structure of the coal deposit can be used to assess the similarity of the geological section. The idea is to calculate the correlation coefficient between two curves that characterize the base surface position of the seam in sections. The calculations based on well-known correlation methods for complex random variables [11]. For correlation coefficient calculation, the position of the seam in the geological section determined with a system of characteristic points coordinated in the conventional coordinate system (figure 2).

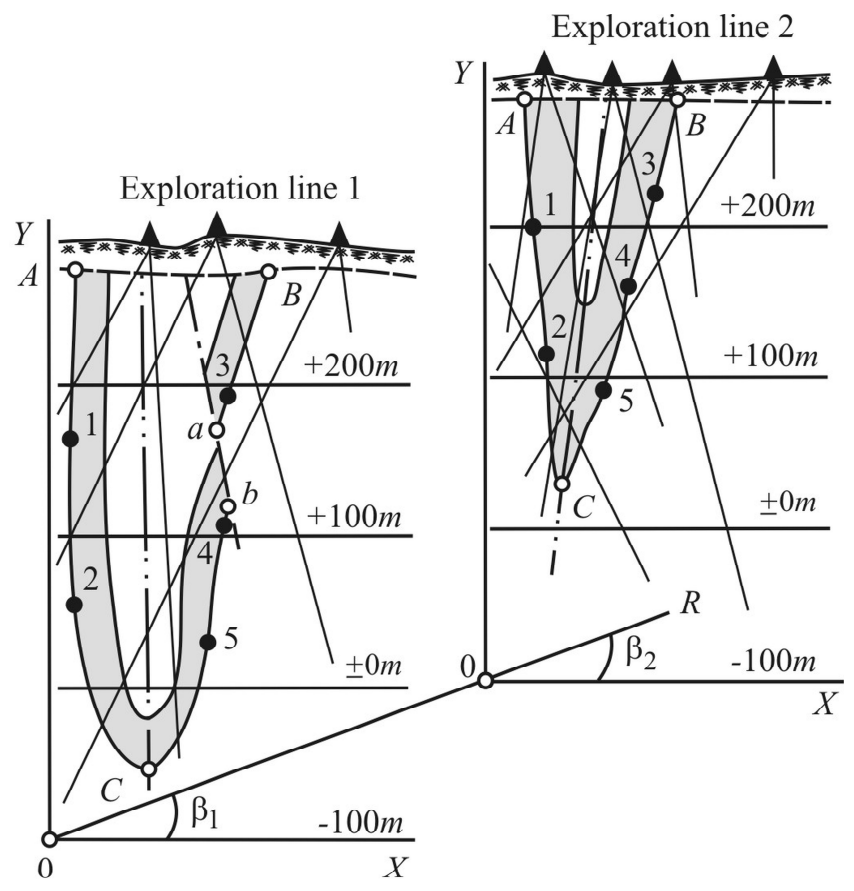

Fig. 2. Selection procedure of characteristic points.

For carrying out an evaluation of the reserves confidence, S. A. Batugin's approach was improved in two ways in order to eliminate the identified limitations.

Firstly, the order of characteristic points selection was changed. The need for separation of characteristic points into three classes was proved.

The class I points include the coal seam outcrops (points $A$ and $B$ in figure 2), the intersection of the seam with the hinge line of the folds (point $C$ in figure 2) and with outskirts of mine.

The class II points include intersection points of the seam with the surface of discontinuity (points of type $a$ and $b$ in figure 2).

Class III points include characteristic points that specify the position of the seam between class I points (points $1-5$ in figure 2). Class III points should be placed evenly between class I points only, and their quantity between pairs of same name points of two analyzed geological section must be the same. Same name points of different sections are assigned the same reference number or letter designation. Same name points are those which refer to the same geological structure, for example, the intersection points of a seam at different sections with the hinge line of the same fold, etc. 
Of course, there could be cases at which the characteristic point doesn't have the same name point on the adjacent geological section.

For each characteristic point, the values of the coordinates $X$ and $Y$ are determined: $x_{1 i}$, $y_{1 i}$ for the point with the number $i$ of the first and $x_{2 i}, y_{2 i}$ for the same name point $i$ of the second section. The origin of the $X O Y$ coordinate system of each section is located on the same defined on the plan axis $R$, which is oriented perpendicular to the preferential direction of the exploration lines.

In case if the angle $\beta_{j}$ between the direction of the exploration line $j$ and the $R$ axis differs from $90^{\circ}$ more than $6^{\circ}$, the measured in the section plane along the $X$ axis coordinates are transformed by multiplying them by $\cos \beta_{j}$.

The correlation coefficient between the coordinates $x_{1 i}$ and $x_{2 i}$ is calculated (only by the characteristic points that are simultaneously present on both sections) as follows:

$$
\tau_{x_{1}, x_{2}}=\frac{\sum_{i=1}^{n}\left(x_{1 i}-\bar{X}_{1}\right)\left(x_{2 i}-\bar{X}_{2}\right)}{\sqrt{\sum_{i=1}^{n}\left(x_{1 i}-\bar{X}_{1}\right)^{2} \sum_{i=1}^{n}\left(x_{2 i}-\bar{X}_{2}\right)^{2}}}
$$

where $\bar{X}_{1}=\frac{\sum_{i=1}^{n} x_{1 i}}{n} ; \bar{X}_{2}=\frac{\sum_{i=1}^{n} x_{2 i}}{n} ; n-$ quantity of characteristic points among all classes, which simultaneously present on both sections.

Analogically, correlation coefficients between $y_{1 i}$ and $y_{2 i}\left(\tau_{y_{1}, y_{2}}\right), x_{1 i}$ and $y_{2 i}\left(\tau_{x_{1}, y_{2}}\right), x_{2 i}$ and $y_{1 i}\left(\tau_{x_{1}, y_{1}}\right)$ are calculated, and based on them the absolute value of correlation coefficient of two complex random variables is calculated (it can have a value in the range from 0 to 2 ):

$$
\left|\tau_{z_{1}, z_{2}}\right|=\sqrt{\left(\tau_{x_{1}, x_{2}}+\tau_{y_{1}, y_{2}}\right)^{2}+\left(\tau_{x_{1}, y_{2}}-\tau_{x_{2}, y_{1}}\right)^{2}},
$$

Secondly, the order of taking into account the appearance or disappearance of characteristic points on the geological section was also changed. This accounting has been proposed to carry out with a correction factor:

$$
\eta=\sqrt{\frac{N-T}{N}}
$$

where $N$ - total number of class I and II characteristic points of the sections, $T$ - total number of class I and II characteristic points present in single section only.

The coefficient of similarity is used as a characteristic that evaluates the degree of sections similarity and calculates as follows:

$$
K_{S}=\eta \frac{\left|\tau_{z_{1}, z_{2}}\right|}{2} .
$$

The use of similarity coefficients is permissible only in case of permissible prediction of seam position between the exploration lines (interpolation permissible). 
For its evaluation, it is supposed to build a "similarity curve" that reflects the relation between the values of the similarity coefficient $K_{S}$ and the distances between geological sections of the exploration lines $L$. The curve is constructed according to the data generated by the method of consecutive rarefaction of the exploration lines grid (similarity coefficients calculated using pairs of adjusted lines, using pairs that "pass" one and two exploration lines). For example, if there are five exploration lines 1, 2, 3, 4 and 5, the similarity coefficients and distances of the following pairs of lines should be used to build the exploration curve: $1-2,2-3,3-4,4-5,1-3,2-4,3-5,1-4$ and $2-5$.

In the presence of interpolation permissible, as the distance between exploration lines $\mathrm{L}$ increases, a decrease in the level of similarity of sections should be observed. However, the similarity coefficients may not depend on the distances $L$. This situation occurs in two cases. Firstly, in a situation where the achieved density of exploration lines is insufficient to disclose the main structural features of the deposit, i.e., for interpolation. Secondly, when working with a field, the nature of the seam location of which does not undergo significant changes (although such a deposit can no longer be fully attributed to the complex located deposits). Separation of these situations is simple: with low complexity of the deposit geological structure, the absolute values of the similarity coefficients should approach to 1 .

Thus, the evaluation of the interpolation permissible and, consequently, the use of similarity coefficients, should be performed using the decision table shown in figure 3 .

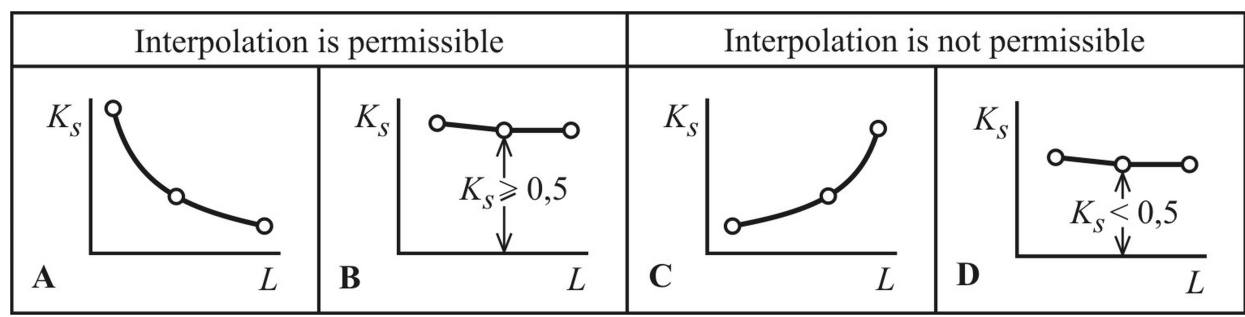

Fig. 3. Decision table for permissible evaluation of the coefficient of similarity use.

\section{Results and conclusions}

Limit values of the similarity coefficient for various categories of resources were experimentally established based on the results obtained by the open-pit process with confirmed reserves categories. It has been established that the most probable value of the similarity coefficient of the cross-sections separating the "inferred" and "indicated" resources is 0.5 (when rounding the coefficient to tenths).

If the "similarity curve" refers to types C or D (figure 3), reserves within the subsoil area shouldn't be allocated, and the obtained geological evidence can only be considered as "Exploration Results".

In the course of work carried out in the interests of Russian and Turkish investors in a number of complex located coal deposits of Kuzbass, it was found that the similarity coefficients of adjusted sections varied from 0.4 to 0.8 . This indicates that the existing approaches to the planning of geological exploration do not fully ensure the possibility of implementing the planned targets for achieving as a result of the reserves exploration the category of "indicated resources".

The previously mentioned high level of non-confirmation of coal reserves $(41 \%)$ in the «Novobachatsky» area took place in a contour in which the similarity coefficients of adjacent sections varied from 0.4 to 0.5 , i.e., could be predicted using the proposed method.

It was established that under conditions of complex located deposits, the error in resources estimates, due to errors in the determination of the average values of seam 
thickness, is significantly (more than twice) inferior in its magnitude to errors of the unreliability of geological structural data. Hence the legitimacy of the selection "inferred" and "indicated" categories based on the similarity coefficients of sections has obtained.

The described method for assessing the confidence of reserves of complex located coal deposits was considered and recommended to use by a decision of the State Commission on mineral resources of Russia and the Society of Russian Experts on Subsoil Use, officially recognized by the CRIRSCO members as a Recognized Professional Organization - RPO. Note that in the Russian classification system applicable for State regulatory purposes to all mineral deposits within the Russian Federation, the division of reserves into classification categories provides for the mandatory use of quantitative approaches (for instance, recommended by OERN).

The obtained results improve the quality of the evaluation of the level of geological confidence of resources and can be used in the analysis of geological information about subsurface and in assessing the geological risk of developing of complex located coal deposits.

\section{References}

1. J. Simensen, J.Perry, J. S. Afr. I. Min. Metall., 99, 321 (1999)

2. O. P. Kreuzer, M. A. Etheridge, AIG News, 100, 20 (2010)

3. J. E. McGill, H. F. J. Theart, S. Afr. I. Min. Metall., 106, 561 (2004)

4. R. Dimitrakopoulos, C. T. Farrelly, M. Godoy, Mining Technology, 111, 1 (2002)

5. The International Template for Reporting of Exploration Results, Mineral Resources and Mineral Reserves (CRISSCO, London, 2013)

6. O. Ashgari, N. M. Esfahani, Arab. J. Geosci. 6, 3 (2013)

7. M. E. Rossi, C. V. Deutsch, Mineral Resource Estimation (Springer, New York, 2013).

8. W. S. Bargawa, N. A. Amri, AIP Publishing, 1705, 1 (2016)

9. V. V. Aksenov, T. B. Rogova, S. V. Shaklein, Applied Mechanics and Materials, 770, 718 (2015)

10.Australian Guidelines for the Estimation and Classification of Coal Resources: (Guidelines Review Committee, Brighton, 2014)

11.P. J. Schreier, L. L. Scharf Statistical signal processing of complex-valued data: the theory of improper and noncircular signals (Cambridge university press, Cambridge, 2010) 